\title{
Comprehensive protection design ideas in the application of Spaceborne Electronic Equipment Components
}

\author{
Feng Qin \\ Beijing Institute of Space Mechanics \& Electricity, Beijing 100094, China \\ 765044951@qq.com
}

Keywords: Spaceborne electronic equipment, Multilayer protection, Electronic components.

\begin{abstract}
The protective measures are very important for the spaceborne electronic equipment to improve the reliability. The purpose of protection is to protect the electronic equipment from Extreme temperature, mechanical impact, electromagnetic interference (EMI), space radiation, electro-Static discharge (ESD) and ground operation damages. In this paper, the principles of electronic protection for spaceborne electronic equipment are reviewed. An integrated protection strategy is suggested, which lists the comprehensive protective design idea in the application of spaceborne electronic equipment components protection to verify the correctness and feasibility of the comprehensive protection design ideas.
\end{abstract}

\section{Introduction}

With the increase of the spaceborne electronic equipment components' intensity, the influence of space environment on the components is increasing, space environment protection design is increasingly important. In response to the temperature, pressure, vibration, shock, radiation, electromagnetic space environment factors, such as thermal protection, EMC, radiation protection reinforcement technology, electrostatic protection, anti-corrosion technology of protection design was put forward. Many scholars of spaceborne electronic devices in a single environment protection are studied, For example, Cheng Gang studied the electrostatic protective design [1],Zhu Jinbiao for heat dissipation structure design, [2],Xu Xiaoting carried out a lot of radiation protection technology research [3],Wang zhixin specifically analyzed the electromagnetic interference [4],Qu Lixin introduced the analysis of the reliability of the circuit board and protection [5],Based on modern protection technology, I comprehensively introduced the various aspects of the electronic equipment protection principle. [6].However often requiring multiple environment of spaceborne electronic equipment, thus an effective comprehensive protective design thinking is particularly important.

Based on the current spaceborne electronic equipment protection theory, this paper puts forward a comprehensive protective design train of thought, shows a design instance of the protective, and finally verifies comprehensive protection design.

\section{Electronic equipment comprehensive protective design ideas}

Protection design must consider the product total life cycle. Spaceborne equipment protection design classification is as follows: According to the (environmental) protection object is divided into vibration impact protection, thermal protection, EMC protection, radiation protection, electrostatic protection, anti-corrosion protection, etc; According to the scope of protection are divided into components protection, veneer protection levels, equipment protection, system protection; According to the technology into shielding isolation, dredge dissipation, shape optimization, performance optimization, redundancy optimization, operation safety, etc; And technical means can be subdivided again a variety of protective measures, such as metal shielding, damping technique, stiffener, adding backup, protection, surface treatment, sealing glue, conductive adhesive or glue, reasonable grounding, reasonable layout, etc.

Comprehensive protection design ideas can briefly for the comprehensive utilization of all kinds of protective means to achieve a variety of protective requirements. The design requirements that first 
we should integrate comprehensive protection needs, teas out all kinds of protective measures, then carry out the protection structure design, validation and optimization, finally conclude a comprehensive method of protection. The basic principle of integrated protection can be summarized as 1) meet the requirements, reliable, measurable (estimated); 2) both plugging and hydrophobic, multiple prevention; 3) the multidisciplinary consideration, multi-angle cuts; 4) modular design, and strive to the most Jane; 5) margin, appropriate redundancy is reasonable; 6) attention to detail design, 7) no additional significant harm.

Comprehensive protection design as a systematic collaborative design, the design process can be briefly as follows: After getting protective performance of the overall index, the project leader need to break out at all levels according to need to the specific requirements of the scope of protection, Send to different positions of the designer, and then respectively to carry out the components level, board level, equipment level of integrated design, system level integrated finally.

\section{Component level comprehensive protective design example}

This section applies the comprehensive protective design ideas for component level the scope of protection design, through to illustrate the feasibility and validity of ideas. The following are Protective performance requirements of the components level, Components is DIP package form; Power consumption is $1 \mathrm{~W}$; Heat resistance is $80{ }^{\circ} \mathrm{C}$; modality analysis $\geq 150 \mathrm{~Hz}$; Under the condition of 15 g, Safety margin Ms $>10$; Under the temperature $20{ }^{\circ} \mathrm{C}$, the conduction path $(0.1 \mathrm{~m}$, with single board level components) heat flow phi acuity $\varphi \geq 10 \mathrm{~W}$; Under the $10 \mathrm{KHz}-1 \mathrm{GHz}$, shielding effectiveness $\geq 30 \mathrm{~dB}$; In irradiation source 1 mev electron flux, eight years in orbit accumulative total dose of radiation can be reduced to $3 \times 10$ rad ( $\mathrm{Si}$ ); $\mathrm{A}$ separate electrostatic protection and anti-corrosion (hot flashes, salt spray, mold) function. By the design requirements can be seen that it is a kind of typical components level comprehensive protection design.

\section{Component level comprehensive protection design}

First of all, from the protection requirements analysis, from the "sealing isolation" and "dredge dissipation" the fundamental point of application of comprehensive protection design basic principle, design refinement into six stages: 1) the protection to achieve sealing and shielding, can meet the electromagnetic compatibility, radiation protection, anti-static, and three requirements; 2) protection should be compatible with thermal protection, best can unblock the heat from the components to the protective structure; 3 ) protection design for solid and reliable, ensure the vibration impact protection; 4) interface is provided for single board level and higher protection scope level; 5) the design feasibility; 6) performance verification and optimization.

The shape optimization, performance optimization, dredge dissipation, and protection to operate a variety of technical means in accordance with the above six steps to carry on the design, finally validated according to various protective environment analysis and optimization.

1) Seal\&block design. First its shape is designed as a cubic shielding box; Take it to install in the shielding box and PCB flexible conductive lap, at the same time increase the screw fastening improve shielding effect. Determine the basic scheme, and then determined using electromagnetic compatibility, radiation protection, static electricity, anti-corrosion protection measures.

Preliminary sure it should include metal shielding case, flexible conductive materials, screw, nut, single ear welding pieces, insulation pads, conductive adhesive, etc. For metal shielding case, In the fight against $10 \mathrm{KHZ}$ - $1 \mathrm{GHZ}$ frequency electromagnetic requirements, it should be Antirust aluminium. The surface uses the conductive anodized and the antistatic coating, Its wall thickness was designed as $1.5 \mathrm{~mm}$, Increasing conductive area, metal shielding shell bottom is designed into flanging form and fixed with screw. Because the conductive gasket has excellent electric properties and shielding performance, but also has good physical and mechanical properties and water vapor sealing performance,we use $\delta 1 \mathrm{~mm}$ conductive gasket as flexible conductive material and Apply a small amount of conductive adhesive in the contact area. As shown in the figure $1 \mathrm{a}$. 
2) Thermal Protection Design. To unblock the heat from components to protective structure, protective structure and components should achieve contact, finally it is concluded that thermal protection measures :using thermal pad ,expanding the cooling area ,increasing the contact pressure ,using thermal conductive sealant ,improving the quality of the surface, etc. So, we increase the entire product with a thermal insulation pad $(\delta 1 \mathrm{~mm})$, which can be compressed down to $0.2 \mathrm{~mm}$. To increase the metal shielding shell heat dissipation area, we increase finned structure metal shielding shell on the surface again. As shown in the figure $1 \mathrm{~b}$.

3) The Vibration Impact Protection. PCB structure strength is low, and the surface quality is not easy to control. So the metal shielding shell flanging contact is easy to form mechanics vulnerabilities and blocking leakage point. So we refer to the shape of the conductive gasket, the glyph structure, into the metal shielding shell and two conductive metal parts, metal shell and the conductive metal is directly on the PCB. As shown in the figure $1 \mathrm{~b}$.

4) Scope of Different Level of Relationship. There are three places need to be coordinated with single board (PCB) level protection design note in the protective design: a) we design a new part named the heat conduction block as the heat exports of the metal shell, which Guide the heat from components to the circuit board frame; b) We need to put single ear welding pieces shall lead to the structure, for implementing electronic grounded; c) PCB and conductive metal frame to decorate a protective layer, and prevent surface wiring. As shown in the figure $1 \mathrm{~b}$.

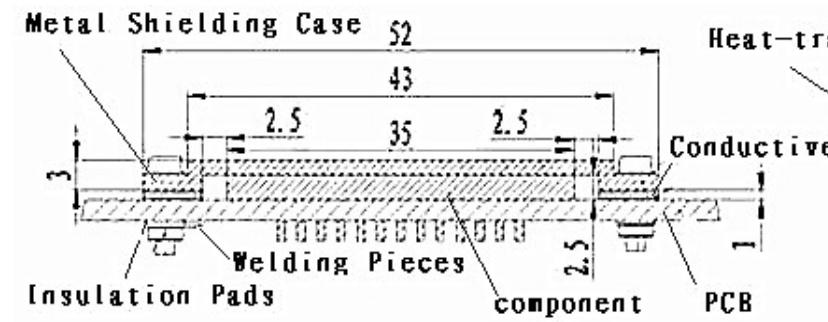

a) Step 1

Fig.1 Protection Design Sketch

5) The Feasibility and Design. Through the processing manufaturability manufacturability, assembly, serviceability and so on to carry on the design review, to ensure the feasibility of the design. This design is feasible, the final design as shown in figure 2.

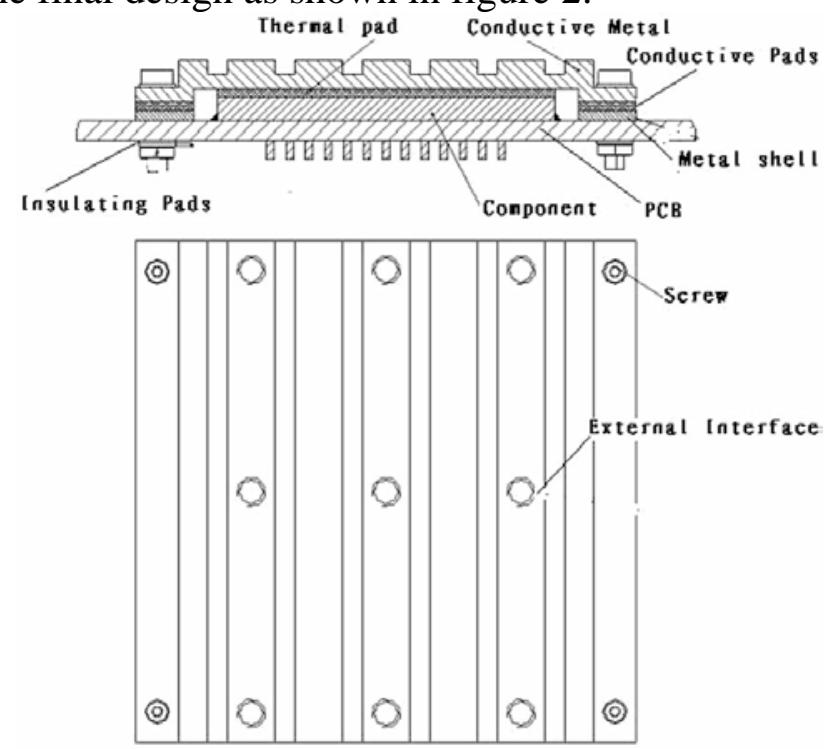

Fig. 2 Component Level Comprehensive Protection Design

6) Performance Verification and Optimization. Preliminary protection design is completed, according to design requirement from mechanical analysis, the cooling effect, electromagnetic shielding effectiveness, anti irradiation effect, electrostatic protection, anti-corrosion design in six aspects, such as protective properties of this structure were tested and optimized. 
a) Mechanical Analysis. This article mainly through modal and quasi static analysis $(\mathrm{X}=\mathrm{Y}=\mathrm{Z}=$ $15 \mathrm{~g})$ verify the structure design of the mechanical properties. Structure occurred in 1 order modal $193 \mathrm{hz}$, fixed holes on a PCB. Quasi static maximum stress $1.44 \mathrm{MPa}$, occurred in the PCB, this is a reasonable margin, three components stress for e $-4 \mathrm{MPa}$, far less than the destruction of the commonly used components. The most severe where X to the PCB surface (vertical direction) on the mechanical analysis. As shown in figure 3 - resistance to learn the results of the analysis.
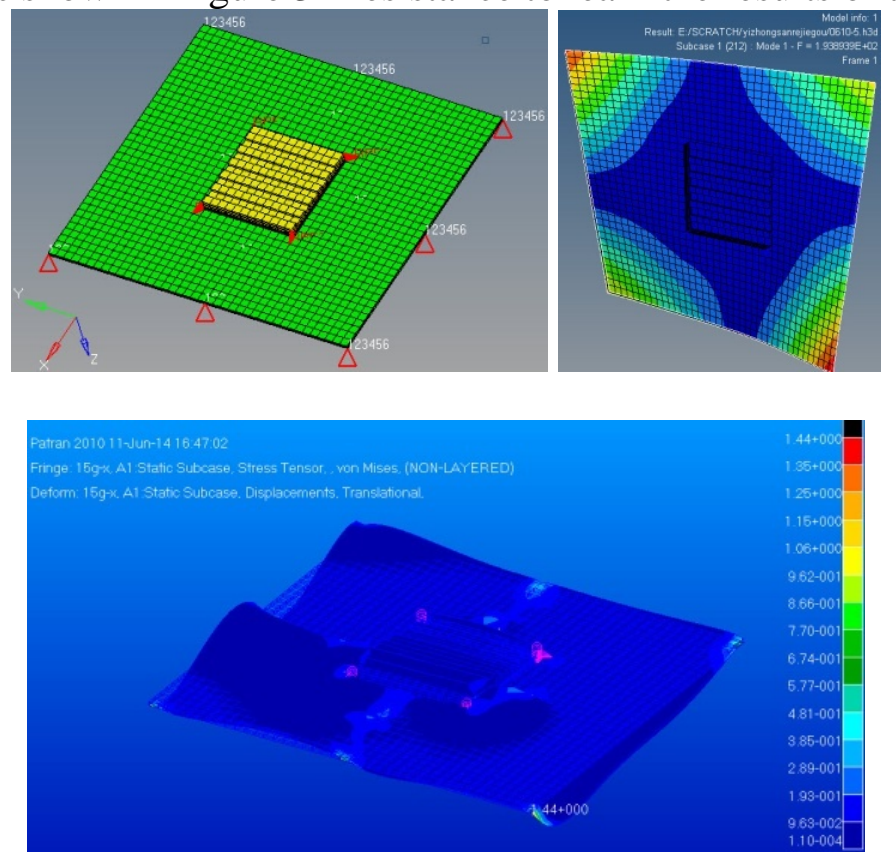

Fig. 3 Resistance to Learn the Results of the Analysis

b) The effect of heat dissipation. Considering the particularity of space environment, we will only verify their heat transfer performance. Heat conduction follow Fourier law:

$$
\phi=-\lambda \mathrm{A} \cdot \frac{d t}{d x}
$$

The phi for the heat flow (W), as the thermal conductivity (), A is the area of the perpendicular to the temperature gradient vector, "' said the direction of heat flow to the low temperature The formula for full contact heat conduction and thermal conductivity of aluminium alloy $125 \mathrm{~W} /\left(\mathrm{m}{ }^{\circ} \mathrm{C}\right), 1 \mathrm{~mm}$ thermal insulation thermal conductivity of $1 \mathrm{~W} /\left(\mathrm{m}{ }^{\circ} \mathrm{C}\right)$, the thermal conductivity of thermal conductivity of insulation transmission path, the $1 \mathrm{~mm}$ thickness direction is only considered to calculate phi is about $240 \mathrm{~W}$, design margin is higher, but is limited by the surface roughness of the contact interface, contact pressure, etc., conduction ability is far lower than the theoretical value, and the effect of heat dissipation with smaller, the result can be accepted.

c) Electromagnetic Shielding Effectiveness. Electromagnetic shielding effectiveness refers to a kind of material to prevent the spread of electromagnetic energy all a measure of the ability. This structure in the metal shell is $1.5 \mathrm{~mm}$, the thinnest place by querying the QJ 1660-89 standard, can be obtained in $10 \mathrm{KHZ}$, wall thickness of $1.5 \mathrm{~mm}$ aluminium alloy shielding effectiveness of $35 \mathrm{db}$, shielding effectiveness in $85 \mathrm{KHZ}$ to $78 \mathrm{db}$, at $1 \mathrm{GHZ}$ is about $50 \mathrm{db}$, shielding effectiveness can be concluded that the structure of shielding effectiveness to meet the design requirements.

d) Irradiation Resistant Effect. Electronic equipment exposed in high-energy particle radiation environment, will have a total dose effect (dar) and single particle effects (SEE) two kinds of effects. Star in the electronic equipment of radiation dose associated with shielding thickness, skin, equipment such as satellite are shielding effect, $1 \mathrm{~mm}$ thick aluminum plate can make the radiation dose was reduced to below 1\%. But because of the shielding material itself has a secondary radiation problem (bremsstrahlung effect), shielding technology can only reduce the total dose of radiation to (2 3) x 10 after rad limits. So in the design of this protection can reduce to $1 \mathrm{~mm}$ thickness, can save the weight and volume of the influence on the structural strength and heat dissipation effect is small. 
e) Electrostatic Protection. Electrostatic damage hazard. Reduce electrostatic loss mainly is to reduce the generation of static electricity, to provide the electrostatic discharge channel. This protection design, the internal and external surface conductive anodized, screening can increase antistatic coating, at the same time at a fixed nut monaural welding, ensure the lap joint resistance $<5$ $\mathrm{m} \Omega$.

f) Anti-corrosion Technology of Protection Design. Damp, salt spray, mold three kinds of environmental factors on the influence on electronic products, and shielding isolation design needs to be done. This section of the protective design mainly four is three steps: material protection, process protection, protective structure, isolation protection. First structural materials using 5 a06 antirust aluminum, all mechanical parts such as screw, nut and so on all use titanium alloy materials; The overall adopts integral enclosed metal box design, joining together the conductive fluid sealant, ability of the three reliable and efficient.

g) Design Optimization and the Theory of Junction. Through the above analysis, the design of components protection design is effective, feasible, single component level protection design requirements and design function and performance, this design meet the requirements, don't have to be optimized, but to make the margin, appropriate wall thickness can be reduced from $1.5 \mathrm{~mm}$ to 1 $\mathrm{mm}$, reduce the impacts on the quality and volume of attached. Through this simple example, prove the feasibility of comprehensive protective design thinking and scientific. In addition it should be pointed out that single board level, equipment level of comprehensive protection design and component level generally consistent and comprehensive protection and system level design is the combination of all three.

\section{Summary}

Electronic equipment reliability is an important performance indicators, in order to satisfy the use requirement to the reliability design of electronic equipment, and protective design is one of the reliability design should follow the basic principles of. This article through to the paper summarizes the existing protection design theory, put forward a comprehensive protective design thinking, through the example, the effective and feasible.

\section{References}

[1] Cheng Gang, Wang xuesong, et al. Space electronics electrostatic protective design [J]. China's individual protective equipment, no.4, 2013.

[2] Zhu Jinbiao, etc. A spaceborne electronic equipment heat dissipation structure design and optimization [J]. Journal of electronic mechanical engineering, 2008, 24 (4) : 11-13.

[3] Xu Xiaoting, etc. Key techniques of spaceborne electronic equipment radiation protection [J]. Journal of mechanical design and theory, xi 'an university of electronic science and technology, 2007.

[4] Wang Zhicheng, etc. Spaceborne electronic equipment test of the three elements of electromagnetic interference analysis [J]. Journal of radio engineering, 39 (6) : 2009-54.

[5] Qu Lixin, etc. Spaceborne electronic equipment reliability of PCB design check [J]. 2010, the Chinese electronic society branch of reliability of the 15th reliability academic conference, 2010, 39-41.

[6] Wang Congsi, Duan Baoyan, etc. Modern electronic equipment protection technology [J]. Journal of electronic mechanical engineering, 2005, 21 (3):1-4. 